\title{
ON THE CAPABILITY OF GROUPS
}

\author{
by GRAHAM ELLIS
}

(Received 19th August 1996)

\begin{abstract}
We show how the third integral homology of a group plays a role in determining whether a given group is isomorphic to an inner automorphism group. Various necessary conditions, and sufficient conditions, for the existence of such an isomorphism are obtained.
\end{abstract}

1991 Mathematics subject classification: $20 \mathrm{~F} 29$.

With regard to the classification of prime-power groups, P. Hall [11] remarked that: "The question of what conditions a group $G$ must fulfil in order that it may be the central quotient group of another group $H, G \cong H / Z(H)$, is an interesting one. But while it is easy to write down a number of necessary conditions, it is not so easy to be sure that they are sufficient." Following [10] we say that a group $G$ is capable if there exists a group $H$ such that $G \cong H / Z(H)$. An account of the basic theory on capability is given in [3]. The capability of finitely generated abelian groups was studied by Baer [1]: such groups are capable if and only if their two highest torsion coefficients agree. In this article we investigate the capability of other classes of groups. Our method involves the third integral homology of a group, and can be applied to any class of groups $G$ for which one can compute the image of a certain homomorphism $\tau: H_{3}(G / Z) \rightarrow Z \wedge G^{a b}$ of abelian groups. Throughout, $Z$ denotes the centre of $G$. The crux of our method is Theorem 4 below. Our method is particularly successful when applied to finitely generated nilpotent groups of class two; Theorem 7 provides a straightforward computation for determining the capability of such groups.

We shall assume that the reader is familiar with the basics of homology of groups (see [12]), and with an exact homology sequence involving a nonabelian exterior product of groups which was obtained (by algebraic methods) in [7] and (by topological methods) in [5]. A self contained algebraic account of this nonabelian exterior product is given in [4].

Let us begin with two propositions on capable groups, the first of which is to be found in [2] (although our proof is slightly different). These provide easily checked criteria for a group not to be capable.

We denote the exponent of a finitely generated abelian group $A$ by $\exp (A)$. We set $\exp (A)=0$ when $A$ contains an element of infinite order, and note that in this case $\exp (A)$ is divisible by all integers. 
Proposition 1 [2]. Let $G$ be a finitely generated capable group. Then every central element $z$ in $G$ has order dividing $\exp \left((G /\langle z\rangle)^{a b}\right)$.

Our second proposition is a more general result. In its statement we denote the order of a group element $x$ by $o(x)$. We set $o(x)=0$ when $x$ is of infinite order. When a central element $z$ in $G$ has been specified, we denote by $\bar{x}$ the image in $(G /\langle z\rangle)^{a b}$ of $x \in G$.

Proposition 2. Let $G$ be a capable group with generating set $J(G)$. Let $z$ be a nontrivial central element in $G$ and let $z^{\prime} \neq 1$ be a (not necessarily proper) power of $z$. Then there exists a generator $x \in J(G)$ such that: (i) $z^{\prime} \neq y^{o(\tilde{x})}$ for all elements $y$ in $Z$; (ii) $\bar{x} \neq a^{o\left(z^{\prime}\right)}$ for all elements $a$ in $(G /\langle z\rangle)^{a b}$; (iii) $z^{\prime}$ is not a power of $x$; (iv) if $G / Z$ is abelian and no element in $J(G)$ has even order then $z^{\prime} \neq w^{o(x)}$ for any $w \in G$.

Although Proposition 2 is not a particularly deep result, it nonetheless has many useful consequences. We deduce a sample of these now. Firstly, note that assertion (i) implies Proposition 1. Assertion (ii) implies that if $G$ is a group whose abelianization $G^{a b}$ is a divisible group and whose centre contains a non-trivial element of finite order, then $G$ is not capable. Assertion (iii) implies the well-known fact that the quaternion group $Q_{2 n}=\left\langle x, y \mid x^{n}=y^{2}, y^{-1} x y=x^{-1}\right\rangle$ of order $4 n, n \geq 2$, is not capable (since its centre is the group of order two generated by $y^{2}$ ). Assertions (iii) and (iv) imply that the group $G=\left\langle x, y \mid x^{9}=y^{3}=1, x y=y x^{4}\right\rangle$ is not capable; this group is of class 2 and order 27, and its centre is generated by $x^{3}$.

Our proof of Proposition 2 involves the tensor product $Z \otimes G^{a b}$ of the centre of $G$ with the abelianization of $G$. (This is the usual tensor product of abelian groups considered as $\mathbb{Z}$-modules.) In fact the proof involves a quotient of the tensor product, namely

$$
Z \wedge G^{a b}=Z \otimes G^{a b} /\langle z \otimes \tilde{z}: z \in Z\rangle
$$

where in this instance $\tilde{z}$ denotes the image in $G^{a b}$ of $z \in Z$. The following easy lemma (whose proof is left to the reader) gives a more computational description of $Z \wedge G^{a b}$.

We shall use additive notation for abelian groups such as $Z \otimes G^{a b}$, and reserve multiplicative notation for groups that are not necessarily abelian.

Lemma 3. Let $J(Z)$ be any generating set for $Z$. The subgroup $\langle z \otimes \tilde{z}: z \in Z\rangle$ of $Z \otimes G^{a b}$ is generated by the elements

$$
\begin{gathered}
x \otimes \tilde{x}, \\
x \otimes \tilde{y}+y \otimes \tilde{x},
\end{gathered}
$$

for $x, y \in J(Z)$. 
In order to give a systematic proof of subsequent results, we introduce a certain subgroup of $Z \wedge G^{a b}$. It is convenient to introduce this now (even though the full details are not needed for our proof of Proposition 2).

We denote by $H_{n}(G)$ the $n$-th homology group of the group $G$ with integer coefficients. Recall from either [5] or [7] that for any group $G$ with centre $Z$ there is a natural exact sequence

$$
H_{3}(G) \longrightarrow H_{3}(G / Z) \stackrel{\imath}{\longrightarrow} Z \wedge G^{a b} \longrightarrow H_{2}(G) \longrightarrow H_{2}(G / Z) \longrightarrow Z \cap[G, G] \longrightarrow 0 .
$$

(Strictly speaking the papers [5] and [7] involve the "non-abelian" exterior product $Z \wedge G$. Since however $G$ and $Z$ act trivially on each other by conjugation, Proposition 2.4 in [5] implies an isomorphism $Z \wedge G \cong Z \wedge G^{a b}$.) The image of the homomorphism $\tau$ is the subgroup of interest.

A characteristic subgroup $Z^{*}(G)$ of $G$ was defined in [2] using the Schur multiplier, and shown to have the property that $Z^{*}(G)$ is trivial if and only if $G$ is capable. We shall call $Z^{*}(G)$ the "epicentre" of $G$, and give an alternative definition of it.

Let us define the epicentre of a group $G$ to be

$$
Z^{*}(G)=\left\{z \in Z \mid z \wedge a \in \operatorname{im}(\tau), \text { for all } a \in G^{a b}\right\},
$$

where $z \wedge a$ denotes a generator of $Z \wedge G^{a b}$. Since $i m(\tau)$ is a subgroup of $Z \wedge G^{a b}$, the set $Z^{*}(G)$ is clearly a (central) subgroup of $G$. The following characterization of capable groups is a "more computational" version of characterizations given in [2], [3] and [9].

Theorem 4. The group $G$ is capable if and only if its epicentre $Z^{*}(G)$ is trivial.

Proof. Recall from [5] (or [7]) that (*) implies an exact sequence

$$
H_{3}(G / Z) \stackrel{\ulcorner}{\longrightarrow} Z \wedge G^{a b} \stackrel{\hookrightarrow}{\longrightarrow} G \wedge G \longrightarrow(G / Z) \wedge(G / Z) \longrightarrow 1
$$

involving the nonabelian exterior square $G \wedge G$. (The definition of this exterior square can be found in [4], [5], or [7]; it involves the conjugation action of $G$ on itself.) $A$ central subgroup of $G$, namely

$$
Z^{\wedge}(G)=\{g \in G: g \wedge x=1 \in G \wedge G \text { for all } x \in G\}
$$

was considered in [9]; it was proved that $G$ is capable if and only if $Z^{\wedge}(G)$ is trivial. Since $\iota(z \wedge \tilde{x})=z \wedge x$ (for all $z \in Z, x \in G)$, we clearly have $Z^{*}(G)=Z^{\wedge}(G)$.

Proof of Proposition 2. Fix a non-trivial element $z \in Z$, and fix some power $z^{\prime} \neq 1$ of $z$. There is a canonical homomorphism 


$$
\phi:(z) \otimes(G /(z))^{a b} \rightarrow Z \wedge G^{a b} .
$$

(If $\bar{y}$ is the image in $(G /\langle z\rangle)^{a b}$ of $\bar{y} \in G^{a b}$, then $\phi(z \otimes \bar{y})=z \wedge \tilde{y}$.) Let us fix for the moment some generator $x \in J(G)$, and let $\bar{x}$ and $\tilde{x}$ denote its images in $(G /\langle z\rangle)^{a b}$ and $G^{a b}$, respectively.

If condition (i) fails for $x$, then the element $z^{\prime} \otimes \bar{x}$ is trivial in $\langle z\rangle \otimes(G /\langle z\rangle)^{a b}$ since for some element $y \in Z$ we have

$$
z^{\prime} \otimes \bar{x}=y^{o(\bar{x})} \otimes \bar{x}=y \otimes \bar{x}^{o(\bar{x})}=y \otimes 0=0 .
$$

Similarly, if condition (ii) fails then $z^{\prime} \otimes \bar{x}$ is trivial. Hence if either (i) or (ii) fails then $z^{\prime} \wedge \tilde{x}=\phi(z \otimes \bar{x})$ is trivial and consequently lies in $i m(\tau)$.

If condition (iii) fails for $x$ then the relation

$$
x^{n} \wedge x=(x \wedge x)^{n}=1
$$

which holds in the nonabelian exterior product $G \wedge G$, implies that $z^{\prime} \wedge x$ is trivial in $G \wedge G$; exactness of the sequence $(* *)$ then implies that $z^{\prime} \wedge \tilde{x}$ lies in $\operatorname{im}(\tau)$.

If condition (iv) fails for $x$ then $G$ is of nilpotency class 2 , and $z^{\prime}=w^{n}$ for some $w \in G$ and $n=o(x)$ where $n$ is odd. The relations

$$
\begin{aligned}
1 & =\left(w^{n} \wedge x\right)\left(x^{n} \wedge w\right)\left([x, w]^{n(n-1) / 2} \wedge x\right)\left([w, x]^{n(n-1) / 2} \wedge w\right) \\
& =\left(w^{n} \wedge x\right)\left(x^{n} \wedge w\right)\left(\left[x^{n}, w^{(n-1) / 2}\right] \wedge x\right)\left(\left[w^{(n-1) / 2}, x^{n}\right] \wedge w\right) \\
& =w^{n} \wedge x \\
& =z^{\prime} \wedge x
\end{aligned}
$$

hold in $G \wedge G$. So (**) implies that $z^{\prime} \wedge \tilde{x}$ lies in $i m(\tau)$.

In conclusion, the epicentre $Z^{*}(G)$ is non-trivial if there exist non-trivial central elements $z$ and $z^{\prime}$ such that at least one of conditions (i)-(iv) fails for each $x \in J(G)$. Proposition 2 thus follows from Theorem 4.

Using Theorem 4 one can attempt to determine those capable groups lying in some class of groups $G$ for which the image of the homomorphism $\tau: H_{3}(G / Z) \longrightarrow Z \wedge G^{a b}$ can be computed.

There are many groups $G$ for which $\operatorname{im}(\tau)=0$. For instance, if $G / Z$ is an aspherical group [6] (such as the free abelian group on two generators, or any knot group, or any other one-relator group whose relator is not a proper power) then $\operatorname{im}(\tau)=0$ since in this case $H_{3}(G / Z)=0$. Alternatively, suppose that $Z$ is a direct summand of $G$; since in this case the surjection $G \longrightarrow G / Z$ is split, the induced homomorphism $H_{3}(G) \rightarrow H_{3}(G / Z)$ is necessarily surjective and consequently $\operatorname{im}(\tau)=0$. The triviality of $\operatorname{im}(\tau)$ is of course equivalent to the surjectivity of the homomorphism $\mathrm{H}_{3}(G) \longrightarrow \mathrm{H}_{3}(G / Z)$. 
Theorem 5. Let $G$ be a group whose centre $Z$ is a direct sum of cyclic groups. Suppose that $Z$ has exponent $\exp (Z)$, and that $\operatorname{im}(\tau)$ is trivial.

(i) If $G^{a b}$ has a cyclic summand $C$ which intersects trivially with the image of $Z$, and if $\exp (Z)$ divides the exponent of $C$, then $G$ is capable.

(ii) If the canonical homomorphism $Z \rightarrow G^{a b}$ maps $Z$ isomorphically onto a direct summand of $G^{a b}$, and if at least two of the cyclic summands of $Z$ have exponent equal to the exponent of $Z$, then $G$ is capable.

Proof. In case (i) let us suppose that $G^{a b}=B \oplus C$. So

$$
\begin{aligned}
Z \wedge G^{a b} & \cong Z \wedge(B \oplus C) \\
& \cong Z \wedge B \oplus Z \wedge C \\
& \cong Z \wedge B \oplus Z \otimes C
\end{aligned}
$$

Suppose that $Z=\oplus_{i}\left\langle x_{i}\right\rangle$ where $x_{i}$ ranges over a set of generators $J(Z)$. Fix any generator $x \in J(Z)$. The generator $c$ of $C$ is such that

$$
0 \neq \lambda x \otimes c \in Z \otimes C \subseteq Z \wedge G^{a b}
$$

whenever $\lambda$ is a positive integer not divisible by $o(x)$. So if $\lambda x$ is the non-trivial projection of some central element $z$ onto the summand $(x)$ of $Z$, then

$$
0 \neq z \wedge c \in Z \otimes C \subseteq Z \wedge G^{a b}
$$

Since $x$ is an arbitrary generator it follows that the epicentre $Z^{*}(G)$ is trivial, and $G$ is capable by Theorem 4 .

In case (ii) the condition that two cyclic summands of $Z$ have exponent equal to the exponent of $Z$ readily yields that

$$
Z^{*}(Z)=\{z \in Z: z \wedge x=0 \in Z \wedge Z \text { for all } x \text { in } Z\}
$$

is the trivial group. But in this case $G^{a b} \cong Z \oplus A$ for some abelian group $A$. Hence

$$
\begin{aligned}
Z \wedge G^{a b} & \cong Z \wedge(Z \oplus A) \\
& \cong Z \wedge Z \oplus Z \otimes A
\end{aligned}
$$

In particular, $Z \wedge Z$ is a subgroup of $Z \wedge G^{a b}$, and so the epicentre $Z^{*}(G)$ must be trivial. Theorem 4 implies that $G$ is capable.

Suppose that $G$ is a finitely generated abelian group. Then $\operatorname{im}(\tau)=0$. So Proposition 1 and Theorem 5(ii) together imply Baer's result [1] that $G$ is capable if and only if its two highest torsion coefficients are equal to $\exp (G)$. 
Suppose that $G$ is the group generated by two elements $x$ and $y$ subject to the relations $x^{2} y=y x^{2}$ and $x^{4}=1$. Then the centre of $G$ is the subgroup of order two generated by $x^{2}$, and the quotient $G / Z$ is isomorphic to the free product $C_{2} * C_{\infty}$. The composite of the canonical surjections $C_{4} * C_{\infty} \longrightarrow G, G \longrightarrow C_{2} * C_{\infty}=G / Z$ induces a surjection $\mathbb{Z}_{4}=H_{3}\left(C_{4} * C_{\infty}\right) \longrightarrow H_{3}\left(C_{2} * C_{\infty}\right)=\mathbb{Z}_{2}$. Consequently the induced homomorphism $H_{3}(G) \longrightarrow H_{3}(G / Z)$ is surjective, and $i m(\tau)=0$. Theorem $5(i)$ implies that $G$ is capable.

Suppose that $G$ is the group generated by four elements $w, x, y$ and $z$ subject to the relations $[w, x]=[w, y]=[w, z]=[x, z]=[y, z]=1$ and $z x=x^{2} y$. The centre of $G$ is generated by $w$ and $z$, and $G / Z$ is aspherical (as it is a one-relator group). Theorem 5 (ii) implies that $G$ is capable.

The capability of a finite nilpotent group $G$ is completely determined by the capability of its Sylow subgroups, thanks to the following proposition.

Proposition 6. Let $G=P_{1} \times \ldots \times P_{n}$ be a direct product of finitely generated groups whose abelianizations $P_{i}^{a b}$ have mutually coprime exponents. Then $G$ is capable if and only if each $P_{i}$ is capable.

Proof. For this proof it is convenient to work with the description of the epicentre

$$
Z^{*}(G)=\{g \in G: g \wedge x=1 \in G \wedge G \text { for all } x \in G\}
$$

given in [9] (and recalled in the above proof of Theorem 4). If $G=G_{1} \times G_{2}$ is a direct product, then from [4] we have an isomorphism

$$
\left(G_{1} \times G_{2}\right) \wedge\left(G_{1} \times G_{2}\right) \cong\left(G_{1} \wedge G_{1}\right) \times\left(G_{2} \wedge G_{2}\right) \times\left(G_{1}^{a b} \otimes G_{2}^{a b}\right)
$$

If in addition the exponents of $G_{1}^{a b}$ and $G_{2}^{a b}$ are coprime, then $G_{1}^{a b} \otimes G_{2}^{a b}$ is trivial and

$$
Z^{*}\left(G_{1} \times G_{2}\right) \cong Z^{*}\left(G_{1}\right) \times Z^{*}\left(G_{2}\right)
$$

We thus have

$$
Z^{*}(G) \cong Z^{*}\left(P_{1}\right) \times \ldots \times Z^{*}\left(P_{n}\right)
$$

and so $Z^{*}(G)$ is trivial if and only if each $Z^{*}\left(P_{i}\right)$ is trivial.

We can compute $\operatorname{im}(\tau)$ for any finitely generated nilpotent group $G$ of class 2 , since the third integral homology of the finitely generated abelian group $G / Z$ is readily determined. For the remainder of this article let us assume that $G$ is such a group; let $J(G, Z)$ be a minimal subset of $G$ whose image modulo $Z$ generates $G / Z$. The computation involves two subgroups $S_{t o r}$ and $S_{j a c}$ of $Z \wedge G^{a b}$. 
For each $x \in G$ set $n(x)=0$ if no power of $x$ lies in $Z$; otherwise let $n(x)$ be the least positive integer such that $x^{n(x)}$ lies in $Z$. Then $S_{\text {tor }}$ is the subgroup generated by the tensors

$$
x^{n(x)} \wedge \tilde{x}
$$

for $x \in J(G, Z)$, and the tensors

$$
\left(w^{n} \wedge \tilde{x}\right)\left(x^{n} \wedge \tilde{w}\right)\left([x, w]^{n(n-1) / 2} \wedge \tilde{x}\right)\left([w, x]^{n(n-1) / 2} \wedge \tilde{w}\right)
$$

for those $w, x \in J(G, Z)$ with $n=n(w)=n(x)$.

The subgroup $S_{j a c}$ is generated by the elements

$$
J(x, y, z)=[x, y] \otimes \tilde{z}+[y, z] \otimes \tilde{x}+[z, x] \otimes \tilde{y}
$$

for $x, y, z \in J(G, Z)$.

The following proposition implies that $S_{t o r}+S_{j a c}$ does not depend on the choice of generating set $J(G, Z)$.

Theorem 7. For any finitely generated group $G$ of nilpotency class 2 , we have

$$
\operatorname{im}(\tau)=S_{t o r}+S_{j a c}
$$

Proof. The abelian group $G / Z$ can be expressed as a direct sum of cyclic groups $G / Z=C_{1} \oplus \ldots \oplus C_{m}$ with $C_{i}$ corresponding to generator $x_{i}$ in $J(G, Z)$. (So $m=|J(G, Z)|$.) Repeated use of the Künneth formula for the homology of a direct sum (see page 233 in [12]) yields an isomorphism $H_{3}(G / Z) \cong A \oplus B \oplus C$, where

$$
\begin{aligned}
& A=\underset{1 \leq i \leq m}{\oplus} H_{3}\left(C_{i}\right), \\
& B=\underset{1 \leq i<j<k \leq m}{\oplus} C_{i} \otimes C_{j} \otimes C_{k}, \\
& C=\underset{1 \leq i<j \leq m}{\oplus} \operatorname{Tor}\left(C_{i}, C_{j}\right) .
\end{aligned}
$$

A careful analysis of the homomorphism $\tau$ shows that $\tau(B)=S_{\text {jac }}$ (cf. [8, Lemma 11]) and that $\tau(A \oplus C)=S_{\text {tor }}$ (cf. [9, Theorem 9]).

The problem of determining the capability of any given finitely generated group of class two can thus be reduced, using Theorems 4 and 7 , to a straightforward computation of finitely generated abelian groups. As an illustration we use these theorems to obtain the following two propositions. 
Proposition 8. Let $G$ be a group of class two. Suppose that $G$ is generated by elements $x$ and $y$, and that $\langle x\rangle \cap Z=\langle y\rangle \cap Z=1$. Then $G$ is capable if and only if $\exp (Z)$ divides $\exp \left(G^{a b}\right)$.

Proof. The subgroup $S_{j a c}$ is trivial since $J(G, Z)=\{x, y\}$ contains just two generators and the identity

$$
\begin{aligned}
J(x, x, z) & =[x, x] \wedge \tilde{z}+[x, z] \wedge \tilde{x}+[z, x] \wedge \tilde{x} \\
& =[x, z] \wedge \tilde{x}-[x, z] \wedge \tilde{x} \\
& =0
\end{aligned}
$$

holds in $Z \wedge G^{a b}$. The subgroup $S_{\text {tor }}$ is trivial since $\langle x\rangle \cap Z=\langle y\rangle \cap Z=1$. Theorem 7 implies that $\operatorname{im}(\tau)$ is trivial. Note that $Z$ is finitely generated. Therefore the proposition follows from Proposition 1 and Theorem 5(i).

Proposition 8 implies for instance that the Burnside group $B(2,3)=\langle x, y| x^{3}=y^{3}=$ $\left.(x y)^{3}=\left(x^{-1} y\right)^{3}=1\right\rangle$ is capable.

Proposition 9. Let $G$ be a finitely generated group of nilpotency class two and of prime exponent. Let $\left\{x_{1}, \ldots, x_{k}\right\}$ be a subset of $G$ corresponding to a basis of the vector space $G / Z$, and suppose that those non-trivial commutators of the form $\left[x_{i}, x_{j}\right]$ with $1 \leq i<j \leq k$ are distinct and constitute a basis for the vector space $[G, G]$. Then $G$ is capable.

Proof. Let $G$ be such a group of class two and exponent $p$. Since its centre $Z$ is of exponent $p$, the commutator subgroup $G^{\prime}$ is a direct summand of $Z$, say $Z=G^{\prime} \oplus A$. Furthermore $A$ is isomorphic to a direct summand of $G^{a b}$, say $G^{a b} \cong A \oplus B$. Note that $A$ may be trivial, whereas $B \cong G / Z$ is a non-trivial elementary abelian group. Now

$$
\begin{aligned}
Z \wedge G^{a b} & \cong G^{\prime} \wedge G^{a b} \oplus A \wedge G^{a b} \\
& \cong G^{\prime} \otimes G^{a b} \oplus A \wedge(A \oplus B) \\
& \cong\left(G^{\prime} \otimes G^{a b}\right) \oplus(A \wedge A) \oplus(A \otimes B)
\end{aligned}
$$

Let $J(G, Z)=\left\{x_{1}, \ldots, x_{k}\right\}$. Since $G$ is of exponent $p$ we have $\langle x\rangle \cap Z=1$ for all $x \in J(G, Z)$. Thus $S_{t o r}$ is the trivial subgroup, and $i m(\tau)=S_{j a c} \subseteq G^{\prime} \otimes G^{a b}$. Since $B \neq 1$, no element of $A$ can lie in the epicentre $Z^{*}(G)$.

Now a basis of $G^{\prime}$ consists of certain non-trivial commutators $\left[x_{i}, x_{j}\right]$ with $x_{i}, x_{j} \in$ $J(G, Z), i<j$. For such a commutator and integer $0<\lambda<p$, the tensor $\lambda[x, y] \otimes x$ is nontrivial in $G^{\prime} \otimes G^{a b} \subseteq Z \wedge G^{a b}$. Moreover, since the commutators $\left[x_{i}, x_{j}\right]$ are linearly independent, the tensor $\lambda[x, y] \otimes x$ does not lie in $S_{j a c}$. It follows from Theorem 7 that $\lambda[x, y]$ does not lie in $Z^{*}(G)$. Consequently $Z^{*}(G)=0$, and $G$ is capable by Theorem 4 . 
Proposition 9 implies that a group $G$ of class two and prime exponent is capable if $G / Z$ is a 2 -generator group. This provides an alternative explanation for the capability of $B(2,3)$.

\section{REFERENCES}

1. R. BAER, Groups with preassigned central and central quotient group, Trans. Amer. Math. Soc. 44 (1938), 387-412.

2. F. R. Beyl, U. Felgner and P. SChmid, On groups occurring as center factor groups, $J$. Algebra 61 (1979), 161-177.

3. F. R. BEYL and J. TAPPE, Groups extensions, representations, and the Schur multiplicator (Lecture Notes in Math. 958, Springer, 1982).

4. R. Brown, D. L. Johnson and E. F. Robertson, Some computations of nonabelian tensor products of groups, J. Algebra 111 (1987), 177-202.

5. R. Brown and J.-L. Loday, Van Kampen theorems for diagrams of spaces, Topology 26 (1987), 311-335.

6. I. M. Chiswell, D. J. Collins and J. HuebschmanN, Aspherical group presentations, Math. Z. 178 (1981), 1-36.

7. G. ElLIS, Nonabelian exterior products of groups and an exact sequence in the homology of groups, Glasgow Math. J. 29 (1987), 13-19.

8. G. Eluis, On five well-known commutator identities, J. Austral. Math. Soc. Ser. A 54 (1993), 1-19.

9. G. Ellis, Tensor products and $q$-crossed modules, J. London Math. Soc. (2) 51 (1995), 243-258.

10. M. HALl and J. K. Senior, The groups of order $2^{n}(n \leq 6)$ (MacMillan, 1964).

11. P. Hall, The classification of prime-power groups, J. Reine Angew. Math. 182 (1940), $130-141$.

12. P. J. Hilton and U. Stammbach, A Course in Homological Algebra, (Graduate Texts in Math. 4, Springer, New York, 1971).

Mathematics Department

UNiversity College Galway

IRELAND

E-mail: graham.ellis@ucg.ie 